\title{
The Pressure Relief System Design for Industrial Reactors
}

\author{
Iztok Hace \\ University of California, 1111 Franklin St., Oakland, CA 94607, USA \\ Correspondence should be addressed to Iztok Hace; iztokhace@gmail.com
}

Received 29 November 2012; Revised 4 June 2013; Accepted 11 September 2013

Academic Editor: Eleonora Bottani

Copyright (C) 2013 Iztok Hace. This is an open access article distributed under the Creative Commons Attribution License, which permits unrestricted use, distribution, and reproduction in any medium, provided the original work is properly cited.

\begin{abstract}
A quick and simple approach for reactor-emergency relief system design-for runaway chemical reactions is presented. A cookbook for system sizing with all main characteristic dimensions and parameters is shown on one realistic example from process industry. System design was done based on existing theories, standards, and correlations obtained from the literature, which were implemented for presented case. A simple and effective method for emergency relief system is shown, which may serve as an example for similar systems design. Obtained results may contribute to better understanding of blow down system frequently used in industrial plants, for increasing safety, decreasing explosion damage, and alleviating the ecological problems together with environmental pollution in case of industrial accidents.
\end{abstract}

\section{Introduction}

In process industry, raw materials are converted into various commercial products using different techniques. One frequently used method is their conversion by exothermic chemical reactions which can lead to a reactor thermal runaway if the heat generation rate exceeds the heat removal rate during process [1]. Pressure build-up during the runaway is caused by an increasing vapor pressure of liquid components and by the production of noncondensable gases. Apart from the loss of reactor inventory due to an uncontrolled conversion process, a runaway reaction may lead to severely damaged equipment or even a physical explosion if pressure build-up inside the reactor exceeds the design pressure. The emergency relief system is composed of vent area, vent rupture membrane, safety relief valve, vent pipes, blow down tank, horizontal condenser, scrubber with absorber and vertical condenser, outflow chimney, corresponding pumps, fan, pipes, fitting, and supply system with electricity, cooling water, and neutralization medium. In case of reaction runaway, the vent rupture disc opens and the reactor mixture blows out into the vent pipes and flows into blow down tank. Due to short residence time of reactor mixture in the blow down tank, the volume changes and the pressure decreases at isothermal conditions, which results in the condensation of reaction mixtures. Remained two phases flow instantaneously blows into horizontal condenser, where it cools down, condensates, and flows into absorber with vertical condenser, where it is neutralized [2-4].

In present study, a detailed design of emergency relief system is shown based on Design Method for Emergency Relief Systems (DIERSs). It incorporates the state-of-theart knowledge obtained from mechanical, electrical, and process engineering based on a long year experiences in the process industries all over the world. All components were designed based on API RP 520 and API STD 526 standards $[5,6]$. In case of problems, reaction runaway may appear due to loss of cooling water in the reactor, overor miss-charged reactant, external fire, and loss of agitation or wrong reaction temperature in reactor. Such cases are difficult to predict, almost not possible to control, and can lead to explosion. Therefore, a proper and correct sized emergency relief system is appropriate method to prevent fatal accidences and environmental pollutions.

The purpose of present study is to

(i) show the main mechanical, electrical, and process fundamentals for this system,

(ii) design emergency relief system with corresponding scrubber, absorption column, and corresponding connections. 


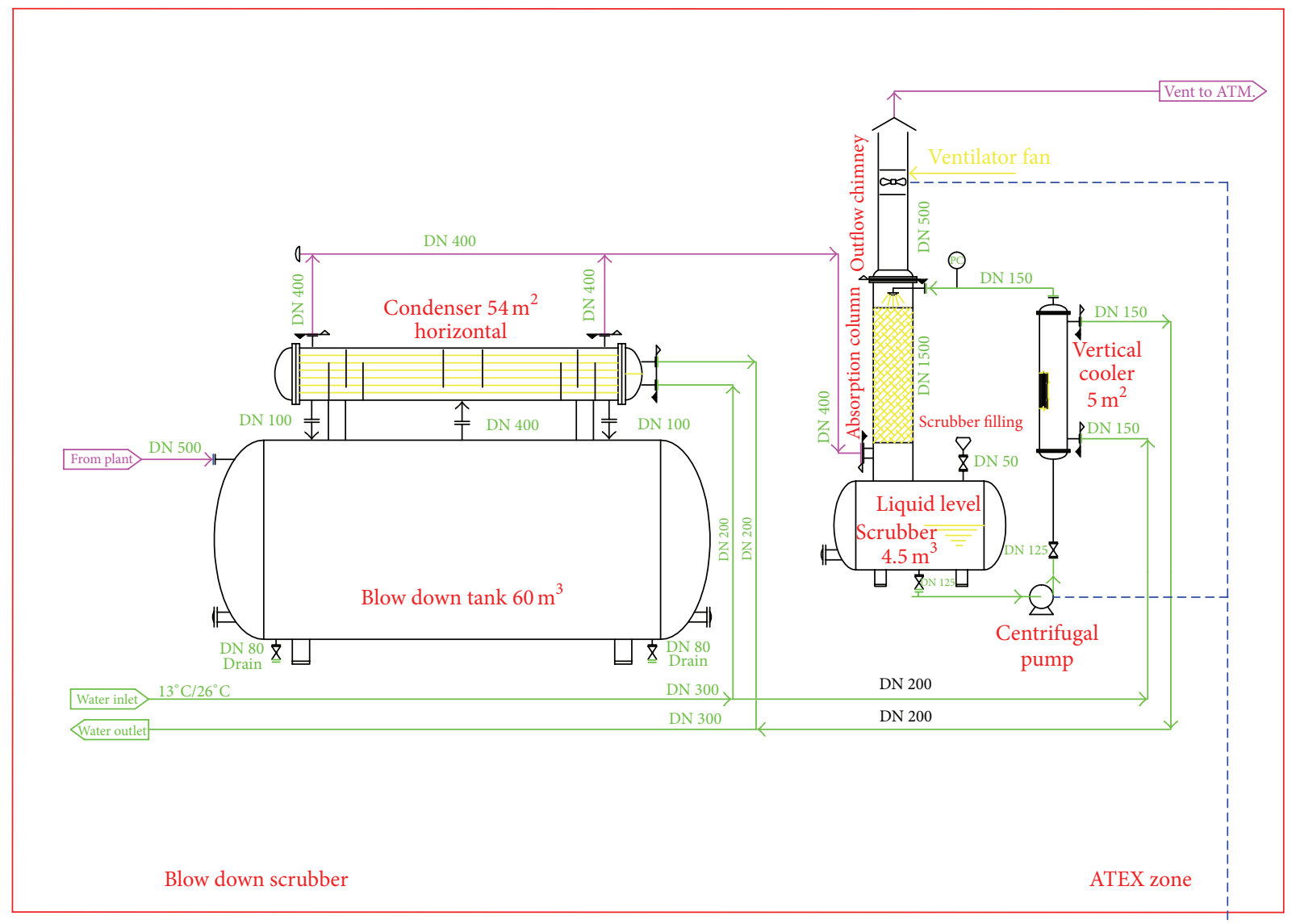

To electric boards located outside ATEX zone

FIGURE 1: Emergency relief system.

Designed emergency relief system is presented in Figure 1. A real industrial example from process industry composed from twelve equal $30 \mathrm{~m}^{3}$ batch reactors equipped with pressure and temperature sensors, cooling and heating mechanism, and rupture discs was taken. In reactors, an exothermic phenol formaldehyde reaction takes place. For reactor cooling towers with capacity of $700 \mathrm{~m}^{3} \cdot \mathrm{h}^{-1}$ of cooling water at $13^{\circ} \mathrm{C} / 26^{\circ} \mathrm{C}$, cooling temperature regime was used.

Based on the reaction runaway the emergency relief system design was made in stages from plant vent system, blow down tank, and scrubber with corresponding heat exchangers and absorption column.

\section{Pressure Relief Devices}

Pressure relief devices design was made according to API RP 520 standard [5]. Detailed pressure relief system on the reactor is presented in Figure 3 and is composed of safety relief valve (SVR) and rupture disc (RD) with dimensions presented in Table 1. The pressure at which SVR piston opens depends on the physicochemical characteristics of reaction system. In present study, it is designed so that SVR opens when the reaction medium pressure is above $70 \cdot 10^{3} \mathrm{~Pa}$. In case of runaway, the outflow medium from reactor blows into vent pipes connected to blow down tank. Very high tip velocities cause a phenomenon known as blow-off where the flame front is lifted and could eventually turn into a blowout. Therefore, determination of the right pipe diameter is important as far as operation of the system is concerned. The vent size is a crucial step at emergency relief system design. In this study, an approach proposed by Fauske [1] and Leung et al. [3] was used. For pressure temperature relation, Antoine equation was used to calculate the vapor pressure at reaction runaway as follows:

$$
P=\exp \left(15.78-\frac{8798}{T}\right) .
$$

The two phase discharge flow rate per unit area was evaluated according to the homogeneous equilibrium model which can be approximated in the low quality region by relation proposed by Leung [2] as follows:

$$
G_{\mathrm{vent}}=0.9 \cdot 144 \cdot \frac{d P}{d T} \cdot\left(\frac{32.2}{778.16} \cdot \frac{T}{c_{\text {pvent }}}\right)^{1 / 2},
$$

where $d P / d T$ was replaced by differential form of (1) and the two phase discharged flow per unit area was evaluated. 
TABLE 1: Calculated system dimensions and characteristics for vent area, horizontal and vertical heat exchanger, blow down and scrubber tank, absorption column, and outflow chimney.

(a)

\begin{tabular}{lccc}
\hline Parameter & Unit & Value & \\
\hline Cooling water data & & & \\
$T_{\text {cin }}$ & ${ }^{\circ} \mathrm{C}$ & 13 & Designed \\
$T_{\text {cout }}$ & ${ }^{\circ} \mathrm{C}$ & 26 & Designed \\
$T_{\text {cout }}$ & ${ }^{\circ} \mathrm{C}$ & 24 & Designed \\
$Q$ & $\mathrm{~m}^{3} \cdot \mathrm{h}^{-1}$ & 700 & Designed \\
Power supply & & $\mathrm{A}$ & \\
$I$ & $\mathrm{~V}$ & 130 & Designed \\
$V$ & $\mathrm{~kW}$ & 380 & Designed \\
$W$ & & 50 & Designed \\
Material flow from reactor & $\mathrm{W}$ & $\mathrm{W}$ & Calculated from (8) \\
$\dot{q}_{c}$ & $\mathrm{~W}$ & Calculated from (8) \\
$\dot{q}_{h}$ & & $2.94 \cdot 10^{6}$ & $2.3 \cdot 10^{6}$ \\
\hline
\end{tabular}

(b)

\begin{tabular}{|c|c|c|c|c|}
\hline Energy released data & & Set condition & Peak condition & \\
\hline$P$ & $\mathrm{~Pa}$ & $2.07 \cdot 10^{5}$ & $2.21 \cdot 10^{5}$ & $\begin{array}{c}\text { Obtained from literature Fauske (1988) } \\
\text { [1], Leung (1986) [2] }\end{array}$ \\
\hline$T$ & $\mathrm{~K}$ & 394.6 & 396.7 & $\begin{array}{c}\text { Obtained from literature Fauske (1988) } \\
\text { [1], Leung (1986) [2] }\end{array}$ \\
\hline$\left(\frac{d T}{d t}\right)_{m},\left(\frac{d T}{d t}\right)_{s}$ & $\mathrm{~K} \cdot \min ^{-1}$ & 15 & 20 & $\begin{array}{c}\text { Obtained from literature Fauske (1988) } \\
\text { [1], Leung (1986) [2] }\end{array}$ \\
\hline$c_{p}$ & $\mathrm{~J} \cdot \mathrm{kg}^{-1} \cdot \mathrm{K}^{-1}$ & 2930.8 & & $\begin{array}{c}\text { Obtained from literature Fauske (1988) } \\
\text { [1], Leung (1986) [2] }\end{array}$ \\
\hline$T_{m}$ & ${ }^{\circ} \mathrm{C}$ & 124.3 & & $\begin{array}{c}\text { Obtained from literature Fauske (1988) } \\
\text { [1], Leung (1986) [2] }\end{array}$ \\
\hline$T_{s}$ & ${ }^{\circ} \mathrm{C}$ & 121.2 & & $\begin{array}{c}\text { Obtained from literature Fauske (1988) } \\
\text { [1], Leung (1986) [2] }\end{array}$ \\
\hline
\end{tabular}

(c)

\begin{tabular}{|c|c|c|c|}
\hline Parameter & Unit & Value & \\
\hline$C_{\text {pcold }}$ & $\mathrm{J} \cdot \mathrm{g}^{-1} \cdot \mathrm{K}^{-1}$ & 4.183 & $\begin{array}{l}\text { Obtained from literature Perry and } \\
\text { Green (2007) [7] }\end{array}$ \\
\hline$C_{\text {phot }}$ & $\mathrm{J} \cdot \mathrm{g}^{-1} \cdot \mathrm{K}^{-1}$ & 2.9281 & $\begin{array}{l}\text { Obtained from literature Perry and } \\
\text { Green (2007) [7] }\end{array}$ \\
\hline \multicolumn{4}{|c|}{ Blow down tank } \\
\hline$V_{o}$ & $\mathrm{~m}^{3}$ & 30 & Designed \\
\hline$V_{\mathrm{bd}}$ & $\mathrm{m}^{3}$ & 60 & Calculated from (6) \\
\hline$P_{\text {sat }}$ & $\mathrm{Pa}$ & $1 \cdot 10^{5}$ & Calculated from (1) \\
\hline$G_{\text {in }}$ & $\mathrm{kg} \cdot \mathrm{s}^{-1}$ & 346 & Calculated from (2) \\
\hline$P$ & $\mathrm{~Pa}$ & $2.07 \cdot 10^{5}$ & $\begin{array}{c}\text { Obtained from literature Fauske (1988) } \\
\text { [1], Leung (1986) [2] }\end{array}$ \\
\hline$P_{1}$ & $\mathrm{~Pa}$ & $1 \cdot 10^{5} \mathrm{~Pa}$ & Calculated from (7) \\
\hline$r_{c}$ & l & 0.24 & Calculated from (34) \\
\hline$G_{\mathrm{HZT}}$ & $\mathrm{kg} \cdot \mathrm{s}^{-1}$ & 82 & Calculated from (35) \\
\hline$W$ & $\mathrm{~m}$ & $25 \cdot 10^{-3}$ & Designed \\
\hline MAWP & $\mathrm{Pa}$ & $2.5 \cdot 10^{5}$ & Designed \\
\hline
\end{tabular}


(c) Continued.

\begin{tabular}{|c|c|c|c|}
\hline Parameter & Unit & Value & \\
\hline \multicolumn{4}{|l|}{ Safety relief valve } \\
\hline $\mathrm{DN}$ & $\mathrm{m}$ & 0.65 & Designed \\
\hline$P_{\text {safety }}$ & $\mathrm{Pa}$ & $70 \cdot 10^{4}$ & Designed \\
\hline \multicolumn{4}{|l|}{ Vent area } \\
\hline$q_{\mathrm{vent}}$ & $\mathrm{J} \cdot \mathrm{kg}^{-1}$ & 846 & Calculated from (6) \\
\hline$G_{\text {vent }}$ & $\mathrm{kg} \cdot \mathrm{m}^{-2} \cdot \mathrm{s}^{-1}$ & 2124 & Calculated from (2) \\
\hline$A_{\text {vent }}$ & $\mathrm{m}^{2}$ & 0.326 & Calculated from (3) \\
\hline$D_{\text {vent }}$ & $\mathrm{m}$ & 0.65 & Calculated from (5) \\
\hline \multicolumn{4}{|l|}{$\begin{array}{l}\text { Calculated data horizontal heat } \\
\text { exchanger }\end{array}$} \\
\hline$L$ & $\mathrm{~mm}$ & 5000 & Designed \\
\hline$h_{\text {DirtIN }}=h_{\text {DirtOUT }}$ & $\mathrm{W} \cdot \mathrm{m}^{-2} \cdot \mathrm{K}^{-1}$ & $698<h<998.7$ & $\begin{array}{l}\text { Obtained from literature Perry and } \\
\text { Green (2007) [7] }\end{array}$ \\
\hline$\lambda_{w}$ & $\mathrm{~W} \cdot \mathrm{m}^{-1} \cdot \mathrm{K}^{-1}$ & 16.2 & $\begin{array}{l}\text { Obtained from literature Perry and } \\
\text { Green (2007) [7] }\end{array}$ \\
\hline$\dot{Q}_{\text {hot }}$ & $\mathrm{W}$ & 585711 & Calculated from (8) \\
\hline$\dot{Q}_{\text {cold }}$ & $\mathrm{W}$ & 366125 & Calculated from (8) \\
\hline$T_{h, \text { out }}$ & ${ }^{\circ} \mathrm{C}$ & 80 & Calculated from (8) \\
\hline LMTD & ${ }^{\circ} \mathrm{C}$ & 76.16 & Calculated from $(9)$ \\
\hline$R_{t}$ & l & 6.83 & $\begin{array}{l}\text { Obtained from literature Richardson et } \\
\text { al. (2009) [10] }\end{array}$ \\
\hline$S$ & l & 0.0594 & $\begin{array}{l}\text { Obtained from literature Richardson et } \\
\text { al. (2009) [10] }\end{array}$ \\
\hline$F_{t}$ & l & 0.96 & $\begin{array}{l}\text { Obtained from literature Richardson et } \\
\text { al. (2009) [10] }\end{array}$ \\
\hline$T_{m}$ & ${ }^{\circ} \mathrm{C}$ & 73.11 & Calculated from (10) \\
\hline $\bar{A}$ & $\mathrm{~m}^{2}$ & 54 & Calculated from (11) \\
\hline$N_{t}$ & / & 85 & Calculated from (13) \\
\hline $\begin{array}{l}\text { Pipe dimensions for horizontal heat } \\
\text { exchanger }\end{array}$ & & & $\begin{array}{l}\text { BWG } 16 \text { Perry and Green (2007) [7], } \\
\text { INOX, AISI316 }\end{array}$ \\
\hline$d_{\text {out }}$ & $\mathrm{mm}$ & 50.8 & \\
\hline$d_{\text {in }}$ & $\mathrm{mm}$ & 46.732 & \\
\hline Wall thickness & $\mathrm{mm}$ & 2.034 & \\
\hline$p_{t}$ & $\mathrm{~mm}$ & 63.5 & Calculated from (14) \\
\hline$D_{b}$ & $\mathrm{~mm}$ & 689.1 & Calculated from (15) \\
\hline $\mathrm{BDC}$ & $\mathrm{mm}$ & 93 & $\begin{array}{l}\text { Obtained from diagrams Richardson } \\
\text { et al. (2009) [10] }\end{array}$ \\
\hline$D_{s}$ & $\mathrm{~mm}$ & 782 & Calculated from (16) \\
\hline$B_{s}$ & $\mathrm{~mm}$ & 313 & Calculated from (17) \\
\hline$A_{s}$ & $\mathrm{~m}^{2}$ & 0.04895 & Calculated from (18) \\
\hline$G_{\text {shell }}$ & $\mathrm{kg} \cdot \mathrm{s}^{-1} \cdot \mathrm{m}^{-2}$ & 1986 & Calculated from (19) \\
\hline$d_{e}$ & $\mathrm{~mm}$ & 36.07 & Calculated from (20) \\
\hline $\mathrm{Re}_{\text {shell }}$ & l & 65127 & Calculated from (21) \\
\hline $\operatorname{Pr}_{\text {shell }}$ & l & 7.48 & Calculated from (22) \\
\hline $\mathrm{Nu}_{\text {shell }}$ & 1 & 323 & Calculated from (23) \\
\hline$h_{\text {shell }}$ & $\mathrm{W} \cdot \mathrm{m}^{-2} \cdot \mathrm{K}^{-1}$ & 5490 & Calculated from (23) and (25) \\
\hline$l_{b}$ & $\mathrm{~mm}$ & 875 & Calculated from (26) \\
\hline$\Delta P_{\text {shell }}$ & $\mathrm{Pa}$ & 53800 & Calculated from (25) \\
\hline$N_{\text {tpp }}$ & 1 & 85 & Calculated from (27) \\
\hline
\end{tabular}


(c) Continued.

\begin{tabular}{|c|c|c|c|}
\hline Parameter & Unit & Value & \\
\hline$G_{\text {tube }}$ & $\mathrm{kg} \cdot \mathrm{m}^{-2} \cdot \mathrm{s}^{-1}$ & 7971 & Calculated from (28) \\
\hline$v_{\text {tube }}$ & $\mathrm{m} \cdot \mathrm{s}^{-1}$ & 2596 & Calculated from (29) \\
\hline $\mathrm{Re}_{\text {tube }}$ & / & 30390 & Calculated from (21) \\
\hline $\operatorname{Pr}_{\text {tube }}$ & / & 0.575 & Calculated from (22) \\
\hline$h_{\text {tube }}$ & $\mathrm{W} \cdot \mathrm{m}^{-2} \cdot \mathrm{K}^{-1}$ & 0.044 & Calculated from (30) \\
\hline$U_{\text {tube }}$ & $\mathrm{W} \cdot \mathrm{m}^{-2} \cdot \mathrm{K}^{-1}$ & 0.022 & Calculated from (32) \\
\hline$U_{\text {shell }}$ & $\mathrm{W} \cdot \mathrm{m}^{-2} \cdot \mathrm{K}^{-1}$ & 1957 & Calculated from (31) \\
\hline$\Delta P_{\text {tube }}$ & $\mathrm{Pa}$ & 91500 & Calculated from (33) \\
\hline \multicolumn{4}{|c|}{ Calculated data vertical heat exchanger } \\
\hline Recycle & $\mathrm{h}^{-1}$ & 22.22 & Calculated from (40) \\
\hline Total heat vertical & $\mathrm{kW}$ & 23553 & Calculated from (36) \\
\hline Heat per recycle & $\mathrm{kW}$ & 145.4 & Calculated from (38) \\
\hline$T_{\text {hinvert. }}$ & ${ }^{\circ} \mathrm{C}$ & 80 & Calculated from (8) \\
\hline $\bar{A}$ & $\mathrm{~m}^{2}$ & 5 & Calculated from (11) \\
\hline$T_{\text {houtvert. }}$ & ${ }^{\circ} \mathrm{C}$ & 40 & Calculated from (8) \\
\hline$T_{\text {cinvert }}$ & ${ }^{\circ} \mathrm{C}$ & 15 & Calculated from (8) \\
\hline$T_{\text {coutvert }}$ & ${ }^{\circ} \mathrm{C}$ & 24 & Calculated from (8) \\
\hline \multicolumn{4}{|c|}{$\begin{array}{l}\text { Pipe dimensions for vertical heat } \\
\text { exchanger }\end{array}$} \\
\hline$L$ & $\mathrm{~mm}$ & 2500 & BWG 16 Perry and Green (2007) [7] \\
\hline$d_{\text {out }}$ & $\mathrm{mm}$ & 50.8 & \\
\hline$d_{\text {in }}$ & $\mathrm{mm}$ & 46.732 & \\
\hline Wall thickness & $\mathrm{mm}$ & 2.034 & \\
\hline$p_{t}$ & $\mathrm{~mm}$ & 63.5 & Calculated from (14) \\
\hline$D_{b}$ & $\mathrm{~mm}$ & 286.7 & Calculated from (15) \\
\hline $\mathrm{BDC}$ & $\mathrm{mm}$ & 88 & $\begin{array}{l}\text { Obtained from diagrams Richardson } \\
\text { et al. (2009) [10] }\end{array}$ \\
\hline$D_{s}$ & $\mathrm{~mm}$ & 388 & Calculated from (16) \\
\hline$B_{s}$ & $\mathrm{~mm}$ & 155.2 & Calculated from (17) \\
\hline$A_{s}$ & $\mathrm{~m}^{2}$ & 0.012043 & Calculated from (18) \\
\hline$G_{s}$ & $\mathrm{~kg} \cdot \mathrm{s}^{-1} \cdot \mathrm{m}^{-2}$ & 8072 & Calculated from (19) \\
\hline$d_{e}$ & $\mathrm{~mm}$ & 36.07 & Calculated from (20) \\
\hline $\operatorname{Re}_{\text {shell }}$ & l & 264718 & Calculated from (21) \\
\hline $\operatorname{Pr}_{\text {shell }}$ & l & 7.48 & Calculated from (22) \\
\hline $\mathrm{Nu}_{\text {shell }}$ & 1 & 2547 & Calculated from (23) \\
\hline$h_{\text {shell }}$ & $\mathrm{W} \cdot \mathrm{m}^{-2} \cdot \mathrm{K}^{-1}$ & 43435 & Calculated from (23) and (24) \\
\hline$l_{b}$ & $\mathrm{~mm}$ & 437.5 & Calculated from (26) \\
\hline$\Delta P_{\text {shell }}$ & $\mathrm{Pa}$ & 82000 & Calculated from (25) \\
\hline$N_{\text {tpp }}$ & 1 & 13 & Calculated from (27) \\
\hline$G_{\text {tube }}$ & $\mathrm{kg} \cdot \mathrm{m}^{-2} \cdot \mathrm{s}^{-1}$ & 6196 & Calculated from (28) \\
\hline$v_{\text {tube }}$ & $\mathrm{m} \cdot \mathrm{s}^{-1}$ & 2018 & Calculated from (29) \\
\hline $\operatorname{Re}_{\text {tube }}$ & I & 23545 & Calculated from (21) \\
\hline $\operatorname{Pr}_{\text {tube }}$ & 1 & 0.575 & Calculated from (22) \\
\hline$\Delta P_{\text {tube }}$ & $\mathrm{Pa}$ & 128000 & Calculated from (33) \\
\hline$h_{\text {tube }}$ & $\mathrm{W} \cdot \mathrm{m}^{-2} \cdot \mathrm{K}^{-1}$ & 0.036 & Calculated from (30) \\
\hline$U_{\text {tube }}$ & $\mathrm{W} \cdot \mathrm{m}^{-2} \cdot \mathrm{K}^{-1}$ & 0.01867 & Calculated from (32) \\
\hline$U_{\text {shell }}$ & $\mathrm{W} \cdot \mathrm{m}^{-2} \cdot \mathrm{K}^{-1}$ & 5617 & Calculated from (31) \\
\hline
\end{tabular}


(c) Continued.

\begin{tabular}{|c|c|c|c|}
\hline Parameter & Unit & Value & \\
\hline LMTD & ${ }^{\circ} \mathrm{C}$ & 38.44 & Calculated from (9) \\
\hline$R_{t}$ & l & 4.44 & $\begin{array}{l}\text { Obtained from literature Richardson et } \\
\text { al. (2009) [10] }\end{array}$ \\
\hline$S$ & l & 0.1385 & $\begin{array}{l}\text { Obtained from literature Richardson et } \\
\text { al. (2009) [10] }\end{array}$ \\
\hline$F_{t}$ & l & 0.96 & $\begin{array}{l}\text { Obtained from literature Richardson et } \\
\text { al. (2009) [10] }\end{array}$ \\
\hline $\mathrm{DT}_{m}$ & ${ }^{\circ} \mathrm{C}$ & 36.7 & Calculated from (10) \\
\hline $\bar{A}$ & $\mathrm{~m}^{2}$ & 5 & Calculated from (11) \\
\hline$N_{t}$ & I & 13 & Calculated from (13) \\
\hline \multicolumn{4}{|l|}{ Absorber and scrubber } \\
\hline \multicolumn{4}{|l|}{ Scrubber } \\
\hline$L_{\mathrm{NaOH}}$ & $\mathrm{kg} \cdot \mathrm{s}^{-1}$ & 42.21 & $\begin{array}{l}\text { Calculated from reaction } \\
\text { stoichiometry }\end{array}$ \\
\hline Recycle & $\mathrm{h}^{-1}$ & 22.22 & Calculated from (40) \\
\hline$V_{\text {scrubber tank }}$ & $\mathrm{m}^{3}$ & 4.5 & Calculated from (40) \\
\hline$w$ & $\mathrm{~m}$ & $10 \cdot 10^{-3}$ & Designed \\
\hline MAWP & $\mathrm{Pa}$ & $1.5 \cdot 10^{5}$ & Designed \\
\hline \multicolumn{4}{|l|}{ Absorber } \\
\hline$(L / G) \cdot\left(\rho_{g} / \rho_{L}\right)^{1 / 2}$ & l & 0.023 & Calculated ratio \\
\hline$\frac{G^{* 2} \cdot F \cdot \mu^{0.1}}{32.2 \cdot \rho_{g} \cdot\left(\rho_{g}-\rho_{L}\right)}$ & l & 1.8 & $\begin{array}{l}\text { Obtained from correlations proposed } \\
\text { in literature Seader et al. (2010) [11] }\end{array}$ \\
\hline$G^{*}$ & $\mathrm{~kg} \cdot \mathrm{m}^{-2} \cdot \mathrm{s}^{-1}$ & 49 & Calculated from obtained factor \\
\hline$A_{\mathrm{abs}}$ & $\mathrm{m}^{2}$ & 1.68 & Calculated from (41) \\
\hline$D_{\text {abs }}$ & $\mathrm{m}$ & 1.46 & Calculated from $(42)$ \\
\hline$\Delta P_{\mathrm{abs}}$ & $\mathrm{m}$ & 0.3048 & $\begin{array}{l}\text { Obtained from literature Seader et al. } \\
\qquad(2010)[11]\end{array}$ \\
\hline No foaming factor $F_{\mathrm{nf}}$ & l & 1 & $\begin{array}{l}\text { Obtained from literature Seader et al. } \\
\qquad(2010)[11]\end{array}$ \\
\hline$F_{\text {IMTP }}$ & I & 12 & $\begin{array}{l}\text { Obtained from literature Seader et al. } \\
\qquad(2010) \text { [11] }\end{array}$ \\
\hline IMTP packing & $\mathrm{mm}$ & 88.9 & $\begin{array}{l}\text { Obtained from literature Seader et al. } \\
\qquad(2010)[11]\end{array}$ \\
\hline$N_{L}$ & l & 1.66 & $\begin{array}{l}\text { Obtained from literature Seader et al. } \\
\qquad(2010)[11]\end{array}$ \\
\hline HETP & $\mathrm{m}$ & 1.83 & $\begin{array}{l}\text { Obtained from literature Seader et al. } \\
\qquad(2010)[11]\end{array}$ \\
\hline$H_{\text {abs }}$ & $\mathrm{m}$ & 3 & $\begin{array}{l}\text { Obtained from literature Seader et al. } \\
\qquad(2010) \text { [11] }\end{array}$ \\
\hline$F_{\text {IMTP }}$ & & 12 & $\begin{array}{l}\text { Obtained from literature Seader et al. } \\
\qquad(2010) \text { [11] }\end{array}$ \\
\hline$L_{\text {operation }}$ & $\mathrm{m}^{3} / \mathrm{h} \cdot \mathrm{m}^{2}$ & 59.9 & $\begin{array}{l}\text { Obtained from literature Seader et al. } \\
\text { (2010) [11] }\end{array}$ \\
\hline$w$ & $\mathrm{~m}$ & $10 \cdot 10^{-3}$ & Designed \\
\hline MAWP & $\mathrm{Pa}$ & $1.0 \cdot 10^{5}$ & Designed \\
\hline \multicolumn{4}{|c|}{$\begin{array}{l}\text { Neutralization reaction in absorber and } \\
\text { in scrubber }\end{array}$} \\
\hline$\Delta H_{\text {react }}$ & $\mathrm{kJ} \cdot \mathrm{mol}^{-1}$ & 22.32 & $\begin{array}{l}\text { Calculated from literature data Perry } \\
\text { and Green (2007) [7] }\end{array}$ \\
\hline$\dot{Q}_{\text {reaction }}$ & $\mathrm{kW}$ & 7.1 & Calculated from (37) \\
\hline
\end{tabular}


(c) Continued.

\begin{tabular}{|c|c|c|c|}
\hline Parameter & Unit & Value & \\
\hline \multicolumn{4}{|l|}{ Centrifugal pump } \\
\hline$L_{\mathrm{NaOH}}$ & $\mathrm{m}^{3} \cdot \mathrm{h}^{-1}$ & 100 & Calculated from reaction stochiometry \\
\hline$\Delta P_{\text {pump }}$ & $\mathrm{Pa}$ & 400000 & Designed \\
\hline \multicolumn{4}{|l|}{ Ventilator fan design } \\
\hline$\Delta P_{\text {Fan }}$ & $\mathrm{Pa}$ & 500 & Calculated from (43) \\
\hline$\emptyset_{V \text { Fan }}$ & $\mathrm{m}^{3} \cdot \mathrm{h}^{-1}$ & 72000 & Calculated from (44) and (45) \\
\hline$v_{r}$ & $\mathrm{~m} \cdot \mathrm{s}^{-1}$ & 230 & Calculated from (47) \\
\hline$\vartheta_{\text {Fan }}$ & RPM & 2920 & Calculated from (44) and (45) \\
\hline$P_{\text {Fan motor }}$ & $\mathrm{kW}$ & 34 & Calculated from (46) \\
\hline$d_{\text {Fan }}$ & $\mathrm{mm}$ & 1460 & Calculated from (44) and (45) \\
\hline \multicolumn{4}{|l|}{ Chimney design } \\
\hline$h_{\text {chimney }}$ & $\mathrm{mm}$ & 6000 & $\begin{array}{l}\text { Obtained from literature diagrams } \\
\text { Bleier (1987) [12] }\end{array}$ \\
\hline$d_{\text {chimney }}$ & $\mathrm{mm}$ & 500 & $\begin{array}{l}\text { Obtained from literature diagrams } \\
\text { Bleier (1987) [12] }\end{array}$ \\
\hline \multicolumn{4}{|l|}{ Pipe sizing } \\
\hline \multicolumn{4}{|l|}{$\begin{array}{l}\text { Pipes from blow down tank via } \\
\text { horizontal heat exchanger to absorber }\end{array}$} \\
\hline \multicolumn{4}{|l|}{$\mathrm{DN}_{\text {pipes }}$} \\
\hline $\begin{array}{l}\text { Blow down tank-horizontal heat } \\
\text { exchanger }\end{array}$ & $\mathrm{mm}$ & 400 & Calculated from (48) \\
\hline Horizontal heat exchanger-absorber & $\mathrm{mm}$ & 400 & Calculated from (48) \\
\hline \multicolumn{4}{|l|}{$\begin{array}{l}\text { Pipes from scrubber via pump and } \\
\text { vertical heat exchanger to absorber }\end{array}$} \\
\hline \multicolumn{4}{|l|}{$\mathrm{DN}_{\text {pipes }}$} \\
\hline Pipes from scrubber via pump & $\mathrm{mm}$ & 125 & $\begin{array}{l}\text { Calculated from literature data Fauske } \\
\text { (1986), Richardson et al. (2009) [3] }\end{array}$ \\
\hline \multicolumn{4}{|l|}{ Cooling water pipes } \\
\hline \multicolumn{4}{|l|}{$\mathrm{DN}_{\text {pipes }}$} \\
\hline Main cooling water pipes & $\mathrm{mm}$ & 300 & $\begin{array}{l}\text { Calculated from literature data Fauske } \\
\text { (1986), Richardson et al. (2009) [3] }\end{array}$ \\
\hline Cooling water pipes after crossing & $\mathrm{mm}$ & 200 & $\begin{array}{l}\text { Calculated from literature data Fauske } \\
\text { (1986), Richardson et al. (2009) [3] }\end{array}$ \\
\hline
\end{tabular}

The analytical vent sizing equation for homogeneous vessel venting is [2-4]

$$
\begin{aligned}
& A_{\text {vent }}= m_{o} \cdot q_{\text {vent }} \\
& \times\left(G _ { \text { vent } } \cdot \left[\left(\frac{V}{m_{o}} \cdot \frac{144}{778.16} \cdot T \cdot \frac{d P}{d T}\right)^{1 / 2}\right.\right. \\
&\left.\left.+\left(c_{\text {pvent }} \cdot \Delta T_{\text {vent }}\right)^{1 / 2}\right]\right)^{-1},
\end{aligned}
$$

where

$$
\begin{gathered}
\Delta T_{\text {vent }}=T_{m}-T_{s}, \\
D=\sqrt{\frac{4 \cdot A_{\text {vent }}}{\pi}}, \\
q_{\text {vent }}=\frac{1}{2} \cdot c_{\text {pvent }} \cdot\left[\left(\frac{d T}{d t}\right)_{s}+\left(\frac{d T}{d t}\right)_{m}\right],
\end{gathered}
$$

and all necessary physical and chemical data are presented as follows.

Physical-chemical parameters use for blow down system design:

$$
\begin{aligned}
& \rho_{\text {liq. }}\left[\mathrm{mol} \cdot \mathrm{dm}^{-3}\right]= \frac{1.3798}{0.31598^{\left(1+(1-(T / 694.25))^{0.32768}\right)},} \\
& c_{p, \text { liq }}\left[\mathrm{J} \cdot \mathrm{Kmol}^{-1} \cdot \mathrm{K}^{-1}\right]=101.720+317.61 \cdot T, \\
& c_{p, g}\left[\mathrm{~J} \cdot \mathrm{Kmol}^{-1} \cdot \mathrm{K}^{-1}\right]= 0.434 \cdot 10^{5}+2.445 \cdot 10^{5} \\
& \cdot\left[\frac{1152 / T}{\sinh [1152 / T]}\right]^{2} \\
&+1.512 \cdot 10^{5} \cdot\left[\frac{507 / T}{\cosh [507 / T]}\right]^{2},
\end{aligned}
$$




$$
\begin{gathered}
\eta_{\text {liq. }}[\mathrm{Pa} \cdot \mathrm{s}]=\exp \left(-43.335+\frac{3881.7}{T}\right. \\
\left.\quad+\frac{4.3983}{\ln (T)}+3.0548 \cdot 10^{24} \cdot T^{-10}\right) \\
\eta_{\text {vap. }}[\mathrm{Pa} \cdot \mathrm{s}]=\frac{1.0094 \cdot 10^{-7} \cdot T^{0.799}}{1+103.1 / T}, \\
\lambda_{\text {vap. }\left[\mathrm{W} \cdot \mathrm{m}^{-1} \cdot \mathrm{K}^{-1}\right]=\frac{0.038846 \cdot T^{0.2392}}{1+985.81 / T+937.17 / T^{2}}} \\
\lambda_{\text {liq. }}\left[\mathrm{W} \cdot \mathrm{m}^{-1} \cdot \mathrm{K}^{-1}\right]=0.18837-0.0001 \cdot T \\
\Delta H_{\text {evap. }}\left[\mathrm{J} \cdot \mathrm{Kmol}{ }^{-1}\right]=7.306 \cdot 10^{7} \cdot\left(1-\frac{T}{694.25}\right)^{0.4246} \\
P_{\text {vapour }}[\mathrm{Pa}]=\exp \left(95.444-\frac{10.13}{T}-10.09 \cdot \ln (T)\right. \\
\left.\quad+6.7603 \cdot 10^{-18} \cdot T^{6}\right)
\end{gathered}
$$

Formation enthalpies (Perry and Green (2007) [7]):

$$
\begin{aligned}
& \Delta H_{\mathrm{NaOH}}^{f}=-469.15 \mathrm{~kJ} \cdot \mathrm{mol}^{-1}, \\
& \Delta H_{\mathrm{PhOH}}^{f}=-165.0 \mathrm{~kJ} \cdot \mathrm{mol}^{-1}, \\
& \Delta H_{\mathrm{H}_{2} \mathrm{O}}^{f}=-285.83 \mathrm{~kJ} \cdot \mathrm{mol}^{-1}, \\
& \Delta H_{\mathrm{NaPhO}}^{f}=-326 \mathrm{~kJ} \cdot \mathrm{mol}^{-1}, \\
& \rho_{50 \mathrm{wt} . \% \mathrm{NaOH}}=1529 \mathrm{~kg} \cdot \mathrm{L}^{-1} .
\end{aligned}
$$

Reaction chemistry at runaway is very complex and depends on the type of used catalyst and other process conditions [14]. In present study, a "worst-case scenario" was used for the thermal runaway of reaction. Design bases of $(d T / d t)_{m}$ and $(d T / d t)_{s}$ were obtained from the literature $[2-4,8]$ and are presented in Table 1. Calculated vent area was compared to the nomogram method from study by Fauske [9] and yield a vent area of $0.326 \mathrm{~m}^{2}$ or $0.65 \mathrm{~m}$ vent area pipe diameter which is in good agreement with the calculated results. Pressure relief devices designs were made according to API RP 526 standard [5]. Detailed presentation of pressure relief devices are presented in Figure 3 with main dimensions shown in Table 1. In present design, it was suggested that RD is located between reactor and SRV. RD protects SRV piston from vapors and opens when tank pressure is above allowable reactor pressure. Maximal pressure which appears at reaction runaway was not known and was obtained from the literature reported data [9]. A design pressure of $2.21 \cdot 10^{5} \mathrm{~Pa}$ was used for system design with $20 \%$ safety factor. In present study, a vent membrane had a pressure relief from -0.75 bar to 0.75 bar. At pressures higher than 0.75 bar, the rupture disc opened and the material was discharged into the blow down tank.

\section{Blow Down Tank Design}

The purpose of the blow down tank is to capture the two phase material flow from reactor and to decrease the pressure of outflow material [9]. Blow down tank design was done based on API RP 520 standard [5]. Blow down tank volume was calculated from the reactor volume as

$$
V_{\mathrm{bd}}=2 \cdot V_{o}
$$

and is reported in Table 1. A factor 2 in (6) was used as engineering safety coefficient which decreases the pressure from reaction runaway by half. It may also be called additional safety margin. The pressure decrease in a blow down tank was calculated by applying the ideal gas behavior at constant temperatures as follows:

$$
\frac{P_{1} \cdot V_{1}}{T_{1}}=\frac{P_{2} \cdot V_{2}}{T_{2}}
$$

and was $1 \cdot 10^{5} \mathrm{~Pa}$. The maximal allowable working pressure (MAWP) for blow down tank was calculated according to API RP 520 standard [5] and was $2.5 \cdot 10^{5} \mathrm{~Pa}$. MAWP was higher than peak pressure at $2.21 \cdot 10^{5} \mathrm{~Pa}$. Due to volume change, the MAWP in blow down tank will not be reached during reaction runaway. In blow down tank, a solid part of two phase runaway material is captured.

\section{Horizontal and Vertical Heat Exchanger Design}

Various procedures for heat exchanger design may be found in the available literature $[9,10,13]$. Only brief calculation procedure is presented $[5,7,10]$.

(i) Assume pipe diameter, length, inside, and outside fouling factor.

(ii) For the pipe construction, an INOX AISI 316 was used with corresponding characteristics presented in Table 1.

(iii) Corresponding energetic balances for horizontal and vertical heat exchanger were written as

$$
\begin{aligned}
\dot{Q} & =\dot{Q}_{\text {hot }}=\dot{Q}_{\text {cold }}=\dot{m}_{c} \cdot c_{p c} \cdot\left(T_{\text {cout }}-T_{\text {cin }}\right) \\
& =\dot{m}_{h} \cdot c_{p h} \cdot\left(T_{\text {hout }}-T_{\text {hin }}\right) .
\end{aligned}
$$

Equation (8) enables calculation of heat duty, $q$, and the hot medium outflow temperatures and results are presented in Table 1.

(iv) The Log Mean Temperature Difference (LMTD) for countercurrent flow was calculated by

$$
\operatorname{LMTD}=\frac{\left(T_{\text {hin }}-T_{\text {cout }}\right)-\left(T_{\text {hout }}-T_{\text {cin }}\right)}{\ln \left(\left(T_{\text {hin }}-T_{\text {cout }}\right) /\left(T_{\text {hout }}-T_{\text {cin }}\right)\right)} .
$$

(v) Based on the experiences and literature data, divided flow shell and even tube passes were assumed for both condensers. Following the procedure proposed by Richardson et al. [10], parameters $R_{t}, S$, and $F_{t}$ were obtained from the corresponding diagrams. Obtained parameters enabled calculation of mean temperature difference:

$$
D T_{m}=F_{t} \cdot \text { LMTD. }
$$


(vi) Overall heat transfer coefficients were assumed from the data reported in the literature [10] within their minimum and maximum values and were between $700 \mathrm{~W} \cdot \mathrm{m}^{2} \cdot \mathrm{K}$ and $1000 \mathrm{~W} \cdot \mathrm{m}^{2} \cdot \mathrm{K}$. Predicted heat transfer coefficients enabled calculation of minimum and maximum provisional area which were taken as average for heat exchanger design:

$$
\bar{A}=\frac{1}{2} \cdot\left(A_{\min }+A_{\max }\right) .
$$

Next, main parameters used for heat exchanger design were calculated using (12)-(18). Pressure drops and the heat transfer coefficients for heat exchanger were calculated using (19)-(25) for the shell and tube site, respectively.

Equations used for horizontal and vertical heat exchanger design are as follows:

$$
\begin{aligned}
& A=\frac{q}{U \cdot D T_{m}}, \\
& N_{t}=\frac{A}{\pi \cdot d_{\text {out }} \cdot L}, \\
& p_{t}=1.25 \cdot d_{o} \text {, } \\
& D_{b}=d_{o} \cdot\left(\frac{N_{t}}{K_{1}}\right)^{1 / n_{1}} \text {, } \\
& D_{S}=D_{b}+\mathrm{BDC} \text {, } \\
& B_{S}=0.4 \cdot D_{S}, \\
& A_{S}=\frac{\left(p_{t}-d_{\text {out }}\right) \cdot D_{S} \cdot B_{S}}{p_{t}}, \\
& G_{\text {shell }}=\frac{\text { shell side flow rate }}{A_{s}}, \\
& d_{e}=\frac{1.10}{d_{\text {out }}} \cdot\left(p_{t}^{2}-0.917 \cdot d_{\text {out }}^{2}\right), \\
& \operatorname{Re}=\frac{d_{e} \cdot \mu \cdot \rho}{\eta}, \\
& \operatorname{Pr}=\frac{\mu \cdot c_{p}}{\lambda}, \\
& \mathrm{Nu}=\frac{h_{\text {shell }} \cdot d_{e}}{k_{f}} \\
& \mathrm{Nu}=j_{h} \cdot \operatorname{Re} \cdot \operatorname{Pr}^{1 / 3} \cdot\left(\frac{\mu}{\mu_{w}}\right)^{0.14} \text {, } \\
& \Delta P_{\text {shell }}=8 \cdot j_{f} \cdot\left(\frac{D_{s}}{d_{e}}\right) \cdot\left(\frac{L}{l_{B}}\right) \cdot\left(\frac{\rho \cdot u_{s}^{2}}{2}\right) \cdot\left(\frac{\mu}{\mu_{w}}\right)^{-0.14} \text {, }
\end{aligned}
$$

$$
\begin{gathered}
l_{B}=\frac{0.7 \cdot L}{4}, \\
N_{\text {tpp }}=\frac{N_{t}}{\text { number of passes }}, \\
G_{\text {tube }}=\frac{G_{\text {in }}}{N_{\text {tpp }} \cdot\left(\pi \cdot d_{\text {in }}^{2} / 4\right)}, \\
\nu_{\text {tube }}=\frac{G_{\text {tube }}}{\rho_{i}} .
\end{gathered}
$$

(vii) The heat transfer coefficient $h_{\text {tube }}$ for turbulent flow was calculated by using next correlation:

$$
h_{\text {tube }}=0.023 \cdot \frac{F_{r}}{d_{\text {in }}} \cdot \operatorname{Re}^{0.8} \cdot \operatorname{Pr}^{0.33} \cdot\left(1+\frac{d_{\text {in }}}{L}\right) \text {. }
$$

(viii) The overall heat transfer factor was calculated on "outside pipes flow":

$$
\begin{aligned}
U_{\text {shell }}=1 \times\left(\frac{1}{h_{\text {shell }}}+\frac{1}{h_{\text {dirtout }}}+\frac{d_{\text {out }}}{d_{\text {in }} \cdot h_{\text {dirtin }}}\right. \\
\left.+\frac{d_{\text {out }}}{d_{\text {in }} \cdot h_{\text {dirtin }}}+\frac{d_{\text {out }} \cdot \ln \left(d_{\text {out }} / d_{\text {in }}\right)}{2 \cdot \lambda_{w}}\right)^{-1} .
\end{aligned}
$$

(ix) The overall heat transfer factor was calculated on "inside pipes flow":

$$
\begin{gathered}
U_{\text {tube }}=1 \times\left(\frac{1}{h_{\text {tube }}}+\frac{1}{h_{\text {dirtin }}}+\frac{d_{\text {in }}}{d_{\text {out }} \cdot h_{\text {dirtout }}}\right. \\
\left.+\frac{d_{\text {in }}}{d_{\text {out }} \cdot h_{\text {shell }}}+\frac{d_{\text {in }} \cdot \ln \left(d_{\text {out }} / d_{\text {in }}\right)}{2 \cdot \lambda_{w}}\right)^{-1} .
\end{gathered}
$$

(x) The tube-side pressure drop was calculated:

$$
\Delta P_{\text {tube }}=\left(1.5+N_{t} \cdot\left[2.5+\frac{8 \cdot j_{f} \cdot L}{d_{\text {in }}}+\left(\frac{\mu}{\mu_{w}}\right)^{-m}\right]\right) \cdot \frac{\rho_{i \cdot v^{2}}}{2} .
$$

Calculated parameters for horizontal and vertical condenser design are reported in Table 1.

\section{Vertical Heat Exchanger Design}

For the vertical heat exchanger design, exactly the same procedure as proposed for the horizontal heat exchanger was used. Firstly, the amount of condensed vapor was calculated from the ratio of vapor pressures at reactor release in the blow down tank and after the volumetric expansion:

$$
r_{c}=\frac{p_{s 1}}{p_{s 2}}
$$

where $p_{s 1}$ and $p_{s 2}$ were the saturated pressures calculated from (1). Obtained $r_{c}$ was used for calculation of gas flow 
which needs to be neutralized and cooled down in vertical heat exchanger:

$$
G_{\mathrm{HZT}}=r_{c} \cdot G_{\mathrm{vent}},
$$

where $G_{\text {HZT }}$ and $G_{\text {vent }}$ are the reactor runaway flow and flow through horizontal condenser reported in Table 1. Calculated $G_{\text {HZT }}$ flow was used for vertical condenser design at the exact same procedure as for horizontal condenser. In the next step, $G_{\text {HZT }}$ flow needed to be cooled down and neutralized in absorption column using sodium hydroxide solution before it was left in the surrounding. $G_{\mathrm{HZT}}$ flows from blow down reactor with temperature which is equal to the temperature of runaway flow due to short residence time. Similar predictions were obtained at design of other blow down processes $[3,8,9]$.

All calculated parameters are presented in Table 1. During $G_{\text {HZT }}$ neutralization reaction, heat was formed. The heat which needs to be cooled down was calculated as the sum of vapor gas heat and the heat produced by neutralization reaction:

$$
\dot{Q}_{\text {total }}=\dot{Q}_{\text {hot gas }}+\dot{Q}_{\text {reaction }} \text {, }
$$

where $\dot{Q}_{\text {react }}$ was calculated using reaction enthalpy as follows:

$$
\dot{Q}_{\text {reaction }}=\Delta H_{\text {reaction }} \cdot G_{\mathrm{HZT}}
$$

and the heat pro recycles using

$$
\dot{Q}_{\text {recycle }}=\frac{\dot{Q}_{\text {total }}}{\text { Recycle }} .
$$

\section{Scrubber Design}

Since the reaction enthalpy for the neutralization reaction of phenol acid with sodium hydroxide cannot be found in the available literature it was calculated from formation enthalpies and was $22.32 \mathrm{~kJ} / \mathrm{mol}$ [10]. Since the composition of the blow down gas is not known exactly, it was assumed that it is composed from phenolic acid [8]. The neutralization reaction of phenolic acid with sodium hydroxide was written as

$$
\mathrm{PhCOOH}+\mathrm{NaOH} \longrightarrow \mathrm{PhCONa}+\mathrm{H}_{2} \mathrm{O}
$$

Neutralization reaction gives the stoichiometric ratio of phenolic acid versus sodium hydroxide and enables the calculation of the amount of neutralization medium needed for neutralization. The necessary $50 \mathrm{wt} . \%$ neutralization liquid flow rate was calculated from the reaction and was $42.3 \mathrm{~kg} / \mathrm{s}$. To decrease the necessary volume of used sodium hydroxide, its recycling was assumed. The recycle flow was calculated using

$$
\text { Recycle }=\frac{G_{\mathrm{HZT}}}{V_{\text {scrubber tank }}} .
$$

The recycle ratio gives scrubber volume of $4.5 \mathrm{~m}^{3}$ and enables the calculation of the liquid and the gas flow rates used for absorption column design. Scrubber design was performed according to API RP 520 standard. All main scrubber dimensions are presented in Table 1. MAWP of scrubber was designed to be $1.5 \cdot 10^{5} \mathrm{~Pa}$.

\section{Absorption Column Design}

Before outflow gas from blow down system was left into surrounding air, it was neutralized by sodium hydroxide in counterflow absorption column. A lot of literature for absorption column design can be found in available literature $[11,14]$. Due to high flow of gas and neutralization fluid the chemical reaction was the limiting step of this process. A generalized approach proposed in the literature was used for column design $[7,11,14]$. Firstly, $(L / G) \cdot\left(\rho_{G} / \rho_{L}\right)^{1 / 2}$ factor was calculated. Pump characteristics limited the pressure drop in absorption column to $1^{\prime \prime}$ water column per feet of packing. Based on existing correlations, factor $\left(G^{* 2} \cdot F \cdot \mu^{0.1}\right) /\left(32.2 \cdot \rho_{g}\right.$. $\left.\left(\rho_{g}-\rho_{L}\right)\right)$ was obtained to be 1.8 and enabled to calculate the gas flux $G^{*}$. A ceramic IMTP packing number 70 was taken as packing material, with main parameters presented in Table 1. Calculated parameters enabled to design an absorption column area and diameter from the following equation:

$$
\begin{aligned}
& G=A \cdot G^{*}, \\
& A=\frac{\pi \cdot D^{2}}{4} .
\end{aligned}
$$

Large absorption column diameter is a consequence of large gas flow at reaction runaway which was designed to be up to $59.5 \mathrm{~m}^{3} \cdot \mathrm{h}^{-1} \cdot \mathrm{m}^{-2}$, which is lower than allowed maximal liquid flow rate of $122 \mathrm{~m}^{3} \cdot \mathrm{h}^{-1} \cdot \mathrm{m}^{-2}$ for IMTO packing number 70 . Next, absorption column height was designed based on the procedure proposed by Seader et al. [11] and was obtained to be $3000 \mathrm{~mm}$. All other column parameters are presented in Table 1 and enabled to design the absorption column pressure drop, which was $30 \cdot 10^{3} \mathrm{~Pa}$.

\section{Ventilator Fan Design}

The vapor gas coming out from absorption column is mainly composed of different phenolic vapors which are heavier than air; therefore, a ventilator fan needs to be inserted into outflow chimney. The role of the fan is to suck the vapor gas coming out from absorption column and to blow it out via chimney into surrounding air. Equations used for the fan design are presented as follows [12].

Design equations for ventilator fan design in outflow chimney.

Pressure of ventilator:

$$
\Delta P_{\text {Fan }}=\rho \cdot g \cdot h_{\text {chimney }}+\frac{\rho \cdot v^{2}}{2} .
$$

Gas vapor velocity:

$$
\emptyset_{V \text { Fan }}=\pi \cdot d_{\text {Fan }} \cdot \vartheta_{\text {Fan }} \cdot A_{\text {Fan }},
$$

where

$$
A_{\mathrm{Fan}}=\frac{\pi \cdot d_{\mathrm{Fan}}^{2}}{4} .
$$

Ventilator motor power:

$$
P_{\text {Fan motor }}=\emptyset_{V \text { Fan }} \cdot \Delta P_{\text {Fan }} .
$$




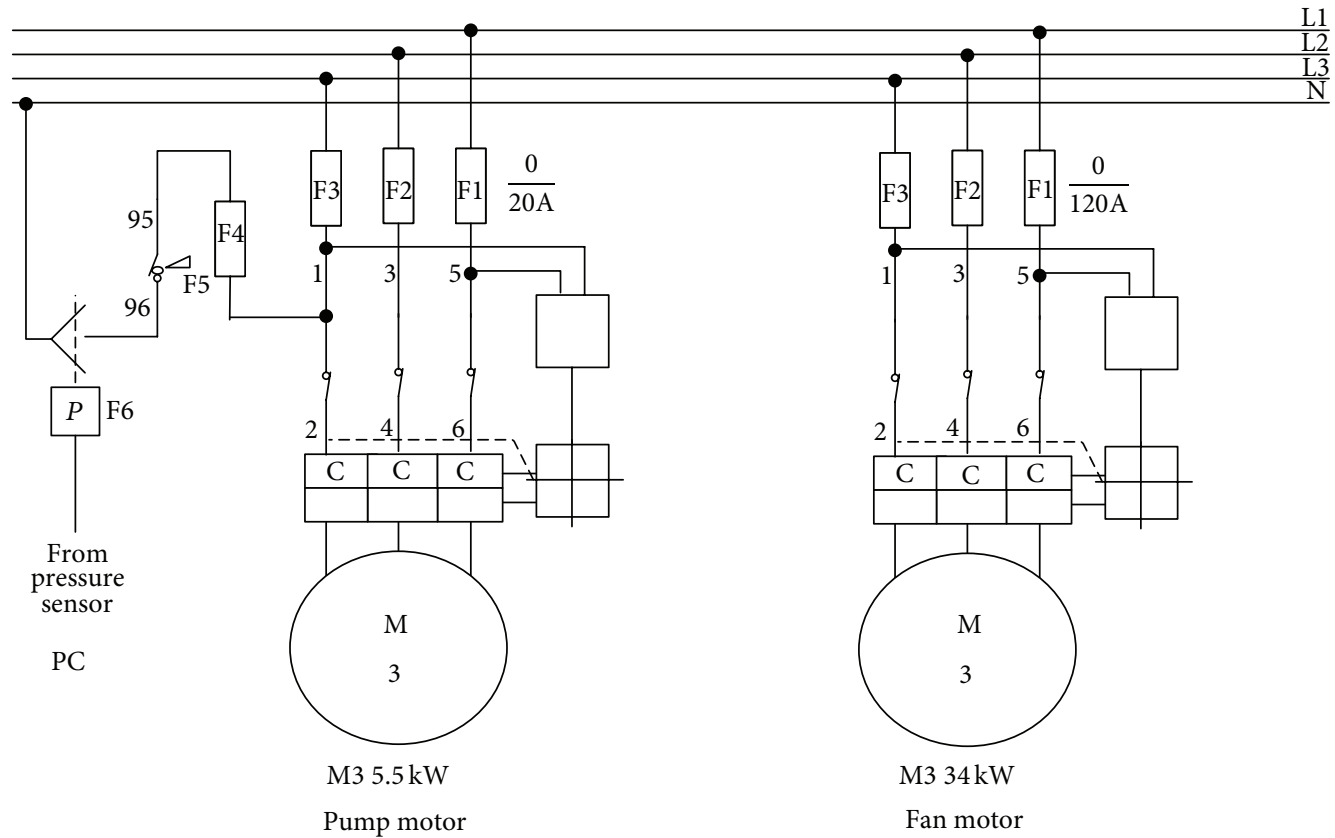

Figure 2: Electrical connections for pump and fan motor.

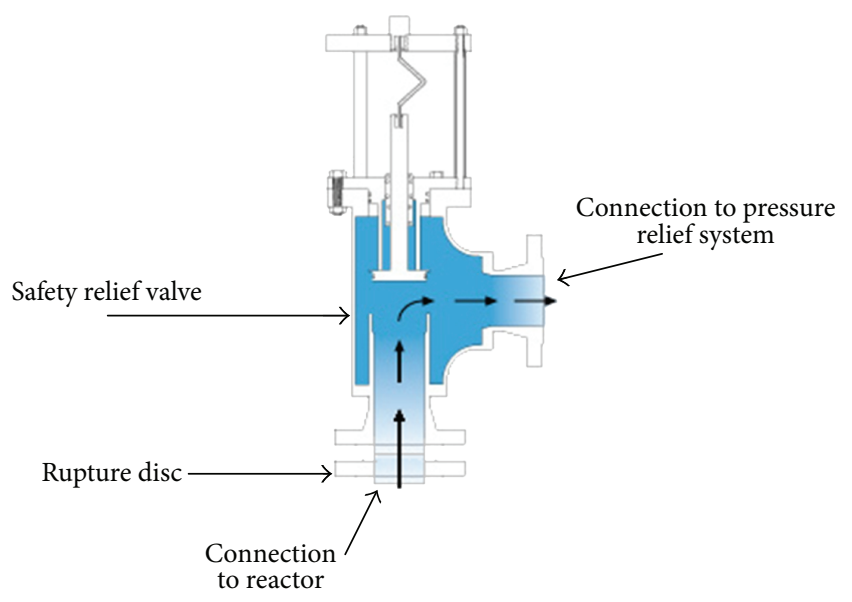

FIGURE 3: Rupture disc and safety relief valve connection to reactor.

Axial fan velocity:

$$
v_{r}=\pi \cdot d_{\mathrm{Fan}} \cdot \vartheta_{\mathrm{Fan}} \cdot
$$

The fan pressure and the fan capacity were designed based on capacity of $82.3 \mathrm{~kg} \cdot \mathrm{s}^{-1}$ of outcoming gas by (43)-(47). Radial velocity of ventilator fan speed $v_{r}$ was calculated from (47) and clearly indicates that an axial ventilator must be applied. All main designed technical characteristics of the ventilator fan are shown in Table 1 . A $34 \mathrm{~kW}$ ventilator fan motor with corresponding ATEX protection with II $2 \mathrm{G} \mathrm{EEx}$ de II B T4 code and motor thermal protection should be used since the motor is located in Ex cone. According to ATEX directive, the electric board with all main connections should be located outside Ex cone which is presented in
Figure 2. Chimney height was obtained from standards and was approximately $6000 \mathrm{~mm}$. All other chimney dimensions are presented in Table $1[4,15]$.

\section{Blow Down and Cooling Water Pipes Design}

Blow down equipment is connected using thick wall pipes made of INOX AISI 316 due to highly corrosive medium, high fluid velocities, and large pressures at reaction runaway. For the vent size pipes, diameter design at reaction runaway frequently used technique proposed by Bleier [12] was used:

$$
D N_{\text {pipe }}=\left(\frac{G}{f_{\text {pipe }}}\right)^{1 / 2} \cdot\left(\frac{\rho}{P}\right)^{1 / 4},
$$

where all parameters are explained in the Nomenclature. The pipe system with $400 \mathrm{~mm}$ pipe diameter was proposed as connection between horizontal heat exchanger and absorption column at given conditions. All other data are presented in Table 1 and Figure 2. Connection pipe system between reactors and blow down tank should be as short as possible, straight with no elbows, no reductions to decrease the amount of condensing flow and to prevent the appearance of plugs which may lead to fatal accidents. Additionally, such system configuration ensures easy maintain and fast pipe cleaning. In parallel, the pipe pressure drops were calculated by method proposed by Crane [16] to ensure smooth and easy flow. The pipe length was designed to be as short as possible with minimal pressure drop which will result in low amount of condensed fluid. High fluid velocities, low friction factors, and minimal pressure drops are expected which agrees with results obtained from the literature [16]. For the cooling water pipes diameter and sodium hydroxide pipe diameter design, a method proposed by Mays [17] which 
is based on the steady state incompressible energy equation utilizing Hazel Williams friction loses and continuity equation was used. The results are presented in Table 1 and in Figure 2.

\section{Pump Design}

Neutralization process characteristics demand $100 \mathrm{~m}^{3} \cdot \mathrm{h}^{-1}$ of $50 \mathrm{wt}$ \% sodium hydroxide. For this, a corresponding pump with $5.5 \mathrm{~kW}$ electrical motor and capacity of $120 \mathrm{~m}^{3} \cdot \mathrm{h}^{-1}$ at approximately $4 \cdot 10^{5} \mathrm{~Pa}$ is proposed. A corresponding electric schema with all relevant parts is presented in Figure 2. Since the pump is situated in Ex cone, a correct motor with motor II 2G EEx de II B T4 code and correct connection according to ATEX standard must be used [18]. Electric board with necessary connections should be located outside the Ex cone. A pressure sensor (PC) is proposed on the pipe from vertical heat exchanger to absorption column. PC has two tasks, to ensure that neutralization medium is pumped all the time from scrubber tank on the absorption column and to prevent the centrifugal pump running dry, which can result in pump failure and in the worst case in pump rotor damage.

It needs to be mentioned that reaction mixture which appears at reaction runaway is very viscous, which may result in appearance of plug in designed pipes, equipment, and huge pressure drops. This problem will be avoided by decreasing the connection pipe length without reductions and low pipe elbow number. Horizontal heat exchanger which is located above the blow down tank decreases the fluid temperature which results in fluid condensation, that is why lower amount of gas need to be neutralized in absorption column. Less required gas for neutralization results in lower $\mathrm{NaOH}$ consumption which decreases operational costs of designed system. Additionally, horizontal heat exchanger produces huge pressure drop which results in lower gas velocities and higher gas/liquid mass transfer coefficients which increases the efficiency of absorption columns [14].

\section{Conclusions}

Designed results of this study demonstrate that proposed method can be used for emergency relief system design. Based on calculated data the following conclusions were made.

Emergency relief system for exothermic reaction for reactor volume up to $30 \mathrm{~m}^{3}$ was designed. A $60 \mathrm{~m}^{3}$ blow down tank, with $700 \mathrm{~m}^{3} \cdot \mathrm{h}^{-1}$ of $13^{\circ} \mathrm{C} / 26^{\circ} \mathrm{C}$ cooling water, and two condensers - horizontal and vertical-with cooling area of $54 \mathrm{~m}^{2}$ and $5 \mathrm{~m}^{2}$ are proposed. An absorber column with diameter of $1.5 \mathrm{~m}$ and $3.0 \mathrm{~m}$ height, $4.5 \mathrm{~m}^{3}$ scrubber tank, and corresponding outflow chimney were designed. All other system parameters are presented in Table 1. It is hoped that the presented procedure will serve as an engineering tool for emergency relief system design in various process industries all over the world.

\section{Nomenclature}

$\begin{array}{ll}L: & \text { Length }(\mathrm{m}) \\ \lambda_{w}: & \text { Thermal conductivity } \\ & \left(\mathrm{W} \cdot \mathrm{m}^{-1} \cdot \mathrm{K}^{-1}\right) \\ C_{\text {pcold }}: & \text { Specific heat of cold medium } \\ & \left(\mathrm{J} \cdot \mathrm{g}^{-1} \cdot \mathrm{K}^{-1}\right) \\ C_{\text {phot }}: & \text { Specific heat of hot medium } \\ & \left(\mathrm{J} \cdot \mathrm{g}^{-1} \cdot \mathrm{K}^{-1}\right)\end{array}$

Cooling water: Specific heat of cooling water

$\dot{Q} \cdot$

$T_{\text {hin }}$ : Temperature of hot medium

$\left({ }^{\circ} \mathrm{C}\right)$

$m_{o}: \quad$ Reactor charge $(\mathrm{kg})$

A: $\quad$ Area $\left(\mathrm{m}^{2}\right)$

$V: \quad$ Reactor volume $\left(\mathrm{m}^{3}\right)$

$\rho: \quad$ Density $\left(\mathrm{kg} \cdot \mathrm{m}^{-3}\right)$

$\dot{q}_{c}$ : $\quad$ Heat release per unit mass cold medium $\left(\mathrm{W} \cdot \mathrm{kg}^{-1}\right)$

$\dot{q}_{h}$ : $\quad$ Heat release per unit mass hot medium $\left(\mathrm{W} \cdot \mathrm{kg}^{-1}\right)$

G: $\quad$ Discharge mass flow rate per

unit area $\left(\mathrm{kg} \cdot \mathrm{m}^{2} \cdot \mathrm{s}^{-1}\right)$

$P: \quad$ Pressure $(\mathrm{Pa})$

$T: \quad$ Temperature $(\mathrm{K})$

$d T / d t: \quad$ Self heat rate in runaway reactions $\left(\mathrm{K} \cdot \mathrm{min}^{1}\right)$

q: $\quad$ Energy release rate $\left(\mathrm{J}^{\mathrm{kgg}}{ }^{-1}\right)$

$\dot{Q}_{\text {hot }}: \quad$ Heat of hot medium (W)

$\dot{Q}_{\text {cold }}$ : $\quad$ Heat of cold medium (W)

$T_{h \text {,out }}$ : Temperature of hot medium

$\left({ }^{\circ} \mathrm{C}\right)$

LMTD: $\quad$ Log mean temperature

difference $\left({ }^{\circ} \mathrm{C}\right)$

$R_{t}$ : $\quad$ Parameter for temperature correction factor prediction

S: $\quad$ Parameter for temperature correction factor prediction Temperature correction factor

$F_{t}:$

$T_{m}$ :

$U$ : $\left({ }^{\circ} \mathrm{C}\right)$

Overall heat transfer coefficient

$\bar{A}: \quad \quad$ Average provisional area $\left(\mathrm{m}^{2}\right)$

$N_{t}: \quad$ Number of tubes

$d: \quad$ Pipe diameter $(\mathrm{mm})$

$r$ : Ration

$\dot{m}: \quad$ Mass flow $\left(\mathrm{kg} \cdot \mathrm{s}^{1}\right)$

$F_{t}: \quad$ Friction factor

$\Delta P_{\text {shell }}$ : $\quad$ Pressure drop on the shell side (Pa)

$\Delta P_{\text {tube }}: \quad$ Pressure drop on the tube side $(\mathrm{Pa})$

BDC: $\quad$ Bundle diameter clearance (mm)

$w: \quad$ Wall thickness $(\mathrm{mm})$

$p_{t}: \quad$ Tube pitch $(\mathrm{mm})$ 


\begin{tabular}{|c|c|c|c|}
\hline$D_{b}:$ & Bundle diameter (mm) & \multirow[t]{3}{*}{$T_{m}:$} & \multirow{2}{*}{$\begin{array}{l}\text { Temperature of reactant } \\
\text { correlates to the temperature }\end{array}$} \\
\hline$D_{s}:$ & Shell diameter (mm) & & \\
\hline$B_{s}:$ & Baffle spacing $(\mathrm{mm})$ & & \multirow{3}{*}{$\begin{array}{l}\text { turn around or maximum over } \\
\text { pressure defined by API RP } \\
520 \text { during runaway (K) }\end{array}$} \\
\hline$A_{s}:$ & Area for cross-flow $\left(\mathrm{m}^{2}\right)$ & \multirow{5}{*}{$T_{s}:$} & \\
\hline$G_{\text {shell }}:$ & Shell-side mass velocity & & \\
\hline & $\left(\mathrm{kg} \cdot \mathrm{s}^{-1} \cdot \mathrm{m}^{-2}\right)$ & & Temperature of reactant \\
\hline$d_{e}:$ & Shell equivalent diameter (mm) & & correlates to set pressure of \\
\hline Re: & Reynolds number & & pressure relief devices $(\mathrm{K})$ \\
\hline Pr: & Prandtl number & $\Delta T:$ & Temperature difference $(\mathrm{K})$ \\
\hline Nu: & Nusselt number & $t:$ & Time $(s)$ \\
\hline$h_{\text {shell }}:$ & Shell-side heat transfer & \multirow{2}{*}{$\begin{array}{l}\eta: \\
\mu:\end{array}$} & Viscosity $(\mathrm{Pa} \cdot \mathrm{s})$ \\
\hline & coefficient $\left(\mathrm{W} \cdot \mathrm{m}^{-2} \cdot \mathrm{K}^{1}\right)$ & & Kinematic viscosity $\left(\mathrm{m}^{2} \cdot \mathrm{s}^{-1}\right)$ \\
\hline$j_{h}$ at $45 \%$ of baffle cut: & Shell-side heat transfer factor & $V:$ & Volume $\left(\mathrm{m}^{3}\right)$ \\
\hline & Baffle spacing $(\mathrm{mm})$ & $D:$ & diameter $(\mathrm{m})$ \\
\hline$N_{\text {tpp }}:$ & Number of tubes per pass & $\rho:$ & Density $\left(\mathrm{kg} \cdot \mathrm{m}^{-3}\right)$ \\
\hline$G_{\text {tube }}:$ & Tube-side mass velocity & $F:$ & Flow reduction factor \\
\hline & $\left(\mathrm{kg} \cdot \mathrm{m}^{-2} \cdot \mathrm{s}^{-1}\right)$ & $V:$ & Voltage (V) \\
\hline$V_{\text {tube }}:$ & Tube-side velocity $\left(\mathrm{m} \cdot \mathrm{s}^{-1}\right)$ & $I:$ & Current (A) \\
\hline $\mathrm{Re}_{\text {tube }}:$ & Inside tube Reynolds number & $W:$ & Power supply (W) \\
\hline $\operatorname{Pr}_{\text {tube }}:$ & Inside tube Prandtl number & GF: & Gas rate $\left(\mathrm{m}^{3} \cdot \mathrm{h}^{-1}\right)$ \\
\hline $\mathrm{Nu}_{\text {tube }}:$ & Inside tube Nusselt number & LF: & Liquid rate $\left(\mathrm{m}^{3} \cdot \mathrm{h}^{-1}\right)$ \\
\hline$j_{f}$ at $45 \%$ of baffle cut: & Shell side friction factor & $c_{\mathrm{Li}}:$ & Liquid concentration $\left(\mathrm{mol} \cdot \mathrm{m}^{3}\right)$ \\
\hline$h_{\text {tubeside }}:$ & Inside heat transfer coefficient & $c_{\mathrm{gi}}:$ & Gas concentration $\left(\mathrm{mol} \cdot \mathrm{m}^{3}\right)$ \\
\hline & $\left(\mathrm{W} \cdot \mathrm{m}^{-2} \cdot \mathrm{K}^{-1}\right)$ & R: & liquid rate to tank volume \\
\hline$U_{\mathrm{i}}:$ & Overall heat transfer factor for & & $\left(\mathrm{h}^{-1}\right)$ \\
\hline & inside tubes flow $\left(\mathrm{W} \cdot \mathrm{m}^{-2} \cdot \mathrm{K}^{-1}\right)$ & $\emptyset_{V \text { Fan }}:$ & Ventilator fan flow $\left(\mathrm{m}^{3} \cdot \mathrm{h}^{-1}\right)$ \\
\hline$U_{\mathrm{o}}:$ & Overall heat transfer factor for & $v_{r}:$ & Axial fan velocity $\left(\mathrm{m} \cdot \mathrm{s}^{-1}\right)$ \\
\hline & outside tubes flow $\left(\mathrm{W} \cdot \mathrm{m}^{-2} \cdot \mathrm{K}^{-1}\right)$ & $\Delta P_{\mathrm{Fan}}:$ & Ventilator fan pressure (Pa) \\
\hline $\bar{U}:$ & Average heat transfer factor & $P_{\text {Fan motor }}:$ & Ventilator motor power (W) \\
\hline & $\left(\mathrm{W} \cdot \mathrm{m}^{-2} \cdot \mathrm{K}^{-1}\right)$ & $\emptyset_{V \text { Fan }}:$ & Rotational velocity of \\
\hline$F_{\text {IMTP }}:$ & IMTP packing factor & & ventilator fan (RPM) \\
\hline$L_{\mathrm{NaOH}}:$ & $\mathrm{NaOH}$ flow $\left(\mathrm{m}^{3} \cdot \mathrm{h}^{1}\right)$ & $A_{\mathrm{Fan}}:$ & Ventilator area $\left(\mathrm{m}^{2}\right)$ \\
\hline Recycle: & Recycle of $\mathrm{NaOH}$ in absorber & $d_{\text {Fan }}:$ & Fan diameter $(\mathrm{mm})$ \\
\hline & $\left(\mathrm{h}^{-1}\right)$ & $h_{\text {chimney }}:$ & Height of outflow chimney \\
\hline$V_{\text {scrubbertank }}:$ & Volume of scrubber tank $\left(\mathrm{m}^{3}\right)$ & & $(\mathrm{mm})$ \\
\hline$A_{\mathrm{abs}}:$ & Absorber area $\left(\mathrm{m}^{2}\right)$ & g: & Gravity acceleration $\left(\mathrm{m}^{2} \cdot \mathrm{s}^{-1}\right)$ \\
\hline$D_{\mathrm{abs}}:$ & Absorber diameter $(\mathrm{m})$ & $v_{\text {vapor }}:$ & Outflow vapor gas velocity \\
\hline$\Delta P_{\mathrm{abs}}:$ & Pressure drop on absorber tray & & $\left(m \cdot s^{1}\right)$ \\
\hline & $(\mathrm{Pa})$ & $f_{\text {pipe }}:$ & Flow reduction factor \\
\hline$F_{\text {IMTP }}:$ & IMTP packing factor & $\Delta H_{\text {react }}:$ & Reaction enthalpy $\left(\mathrm{J} \cdot \mathrm{mol}^{1}\right)$ \\
\hline IMTP packing: & Mark of absorber fill & $G^{*}:$ & Gas flux $\left(\mathrm{kg} \cdot \mathrm{s}^{1} \cdot \mathrm{m}^{2}\right)$ \\
\hline$N_{L}:$ & Number of theoretical plates or & DN: & Inner diameter (m) \\
\hline & trays & bd: & Blow down \\
\hline HETP: & Height equivalent to the & $m:$ & Pressure turnaround, mean \\
\hline & theoretical plate $(\mathrm{m})$ & $\max .:$ & Maximal value \\
\hline$H_{\mathrm{abs}}:$ & Height of absorption column & $s:$ & Relief set conditions, shell \\
\hline & $(\mathrm{m})$ & $o:$ & Initial conditions \\
\hline$V_{\mathrm{bd}}:$ & Blow down volume $\left(\mathrm{m}^{3}\right)$ & cin: & Cold in \\
\hline$V_{\text {max }}:$ & Maximal tank volume $\left(\mathrm{m}^{3}\right)$ & cout: & Cold out \\
\hline$q_{\text {vent }}:$ & Heat release per unit mass at & abs: & Absorber \\
\hline & reaction runaway $\left(\mathrm{W} \cdot \mathrm{s}^{-1}\right)$ & vent: & Vent \\
\hline$c_{p}:$ & Specific heat of material & BWG: & Birmingham Wire Gauge \\
\hline & $\left(\mathrm{J} \cdot \mathrm{g}^{-1} \cdot \mathrm{K}^{-1}\right)$ & dirt in/dirt out: & Coatings of dirt on the in and \\
\hline$(d T / d t)_{m}:$ & Self heat rate at temperature & & out site of pipe \\
\hline & turn around or maximal over & $w:$ & Wall \\
\hline & pressure defined by API RP 520 & tube: & Tube side \\
\hline & during run away $\left(\mathrm{K} \cdot \mathrm{s}^{-1}\right)$ & shell: & Shell side \\
\hline$(d T / d t)_{s}:$ & Self heat rate at set pressure of & $i, j:$ & Refers to tube and shell side \\
\hline & pressure relief devices $\left(\mathrm{K} \cdot \mathrm{s}^{-1}\right)$ & & \\
\hline
\end{tabular}




$\begin{array}{ll}\text { 1, 2, 3: } & \text { Refers to position } \\ \text { S1, S2: } & \text { Saturated pressure at position } 1 \\ & \text { and } 2 \\ \text { HZT: } & \text { Horizontal } \\ \text { hin: } & \text { Hot in } \\ \text { hout: } & \text { Hot out } \\ \text { out: } & \text { Out site } \\ :: & \text { Unit in time } \\ e: & \text { Equivalent } \\ \text { IMTP: } & \text { IMTP packing type } \\ c: & \text { Cold } \\ h: & \text { Hot } \\ t: & \text { Pitch } \\ b: & \text { Bundle } \\ h: & \text { Heat } \\ f: & \text { Friction } \\ i: & \text { Inside } \\ o: & \text { Outside } \\ \text { abs: } & \text { Absorber } \\ L: & \text { Theoretical } \\ \pi: & \text { 3.14 } \\ s: & \text { Set point } \\ \text { PE: } & \text { Electrically grounded } \\ \text { U1, U2: } & \text { Electro phases } \\ \text { V1, V2: } & \text { Electro phases } \\ \text { W1, W2: } & \text { Electro phases } \\ \text { TK: } & \text { Electro phases } \\ \text { L1, L2, L3: } & \text { Electro phases } \\ N: & \text { Neutral phase } \\ \text { S1: } & \text { Switch 1 } \\ 0,1,2,3,4: & \text { Position in switch and } \\ \text { F1: } & \text { correction } \\ \text { AC3: } & \text { Fusion } 1 \\ \text { X1,X2: } & \text { Analog current } \\ \text { M: } & \text { Contactor } \\ 3 \sim, \Lambda / \Delta: & \text { Three phase electro motor } \\ \text { MAWP: } & \text { Maximal allowed working } \\ & \text { pressure. } \\ & \end{array}$

\section{References}

[1] H. K. Fauske, "Emergency relief system design for reactive and non-reactive systems: extension of the DIERS methodology," Plant/Operations Progress, vol. 7, no. 3, pp. 153-158, 1988.

[2] J. C. Leung, "Simplified vent sizing equations for emergency relief requirements in reactors and storage vessels," AIChE Journal, vol. 32, no. 10, pp. 1622-1634, 1986.

[3] J. C. Leung, H. K. Fauske, and H. G. Fisher, "Thermal runaway reactions in a low thermal inertia apparatus," Thermochimica Acta, vol. 104, pp. 13-29, 1986.

[4] R. C. Rosaler, Standard Handbook of Plant Engineering, McGraw-Hill, New York, NY, USA, 3rd edition, 2002.

[5] API RP 520, Sizing, Selection and Installation of Pressure Relieving Devices in Refineries, American Petroleum Institution, 2000.

[6] API STD 526, Flanged Steel Pressure Relief Valves, American Petroleum Institution, 6th edition, 2012.

[7] R. H. Perry and D. W. Green, Perry's Chemical Engineers' Handbook, McGraw-Hill, New York, NY, USA, 2007.
[8] J. L. Gustin, J. Fillion, G. Tréand, and K. E. Biyaali, “The phenol + formaldehyde runaway reaction. Vent sizing for reactor protection," Journal of Loss Prevention in the Process Industries, vol. 6, no. 2, pp. 103-113, 1993.

[9] H. K. Fauske, "Generalized vent sizing monogram for runaway chemical reactions," Plant/Operations Progress, vol. 3, no. 4, pp. 213-215, 1984.

[10] J. F. Richardson, J. H. Harker, and J. R. Backhurst, Coulson and Richardson's Chemical Engineering, Butterworth-Heinemann, Boston, Mass, USA, 2009.

[11] J. D. Seader, E. J. Henley, and D. K. Roter, Separation Process Principles, John Wiley \& Sons, New York, NY, USA, 2010.

[12] F. Bleier, Fan Handbook: Selection, Application and Design, McGraw-Hill, New York, NY, USA, 1987.

[13] S. A. Kudchadker, A. P. Kudchadker, R. C. Wilhoit, and B. J. Zwolinski, "Ideal gas thermodynamic properties of phenol and cresols," Journal of Physical and Chemical Reference Data, vol. 7, no. 2, pp. 417-423, 1978.

[14] I. Hace, "Comparison of concentration profiles for boundary layers in absorption with chemical reaction processes," Chemical Engineering and Technology, vol. 29, no. 5, pp. 574-582, 2006.

[15] J. C. Leung and H. K. Fauske, "Runaway system characterization and vent sizing based on DIERS methodology," Plant/Operations Progress, vol. 6, no. 2, pp. 77-83, 1987.

[16] Crane Technical Paper, “A Process Design Engineer's Perspective on Using Equivalent Lengths of Valves and Fittings in Pipeline Pressure Drop Calculations, 410(TP-410)," 2009.

[17] L. W. Mays, Hydraulic Design Handbook, McGraw-Hill, New York, NY, USA, 1999.

[18] “ATEX Directive 94/9EC' on equipment and protective systems intended for use in potentially explosive atmospheres (ATEX)," http://ec.europa.eu/enterprise/sectors/mechanical/documents/ legislation/atex/. 

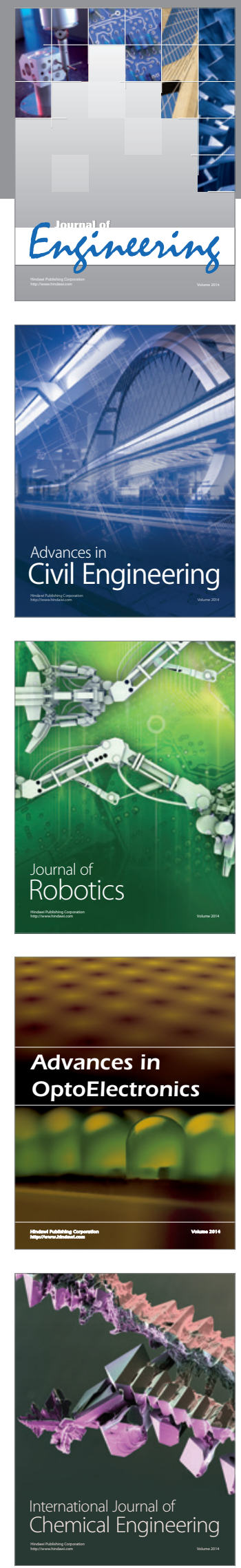

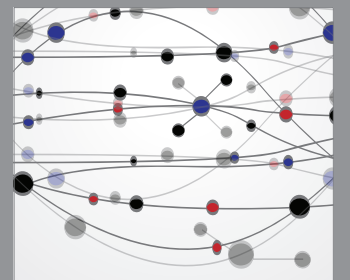

The Scientific World Journal
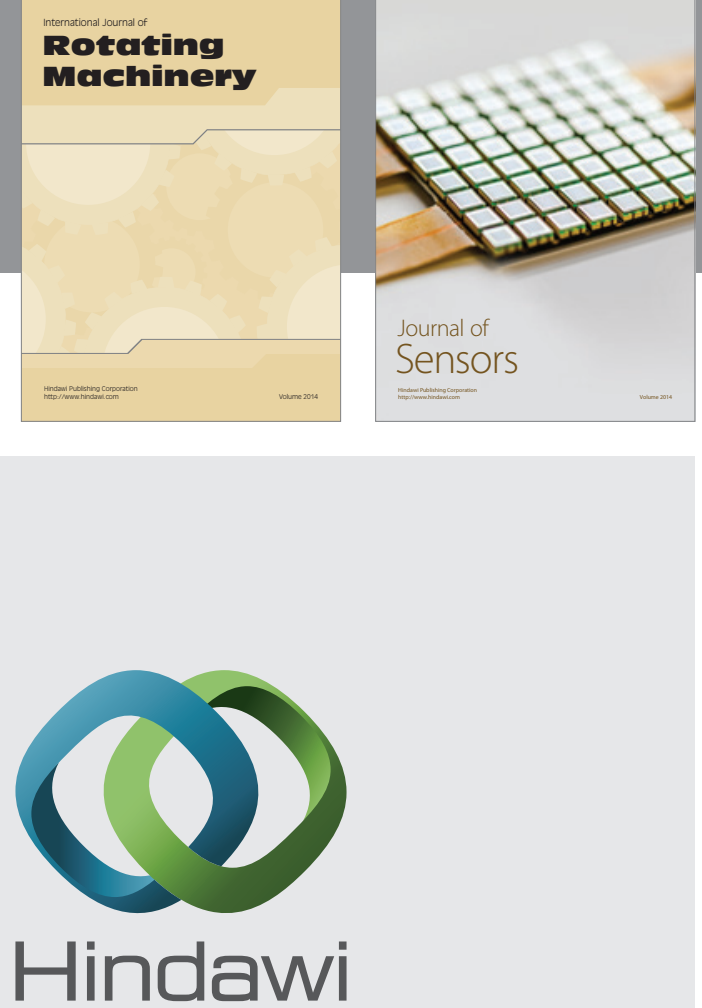

Submit your manuscripts at http://www.hindawi.com
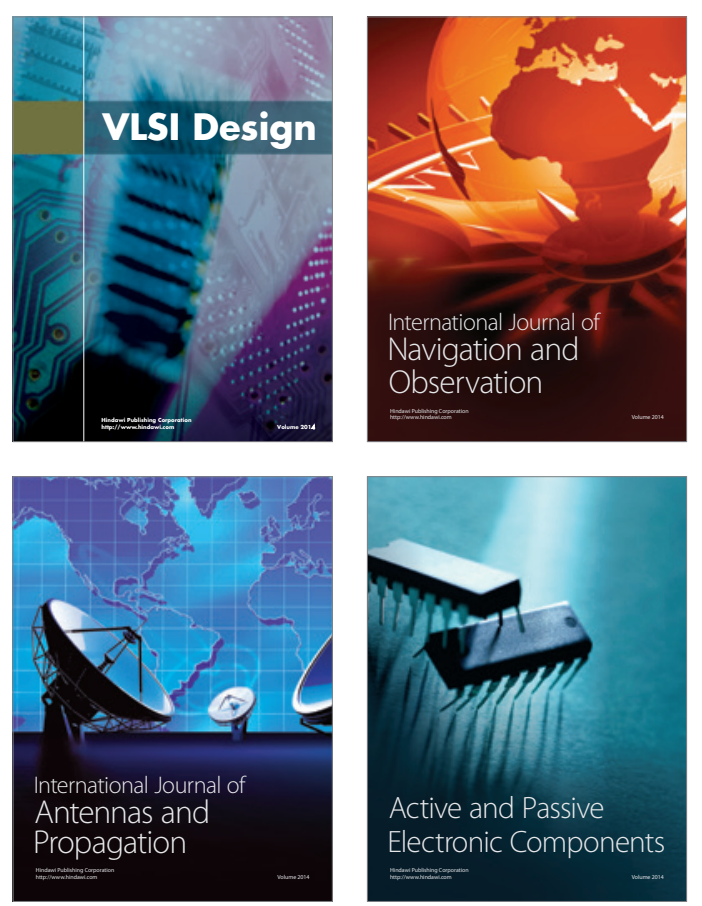
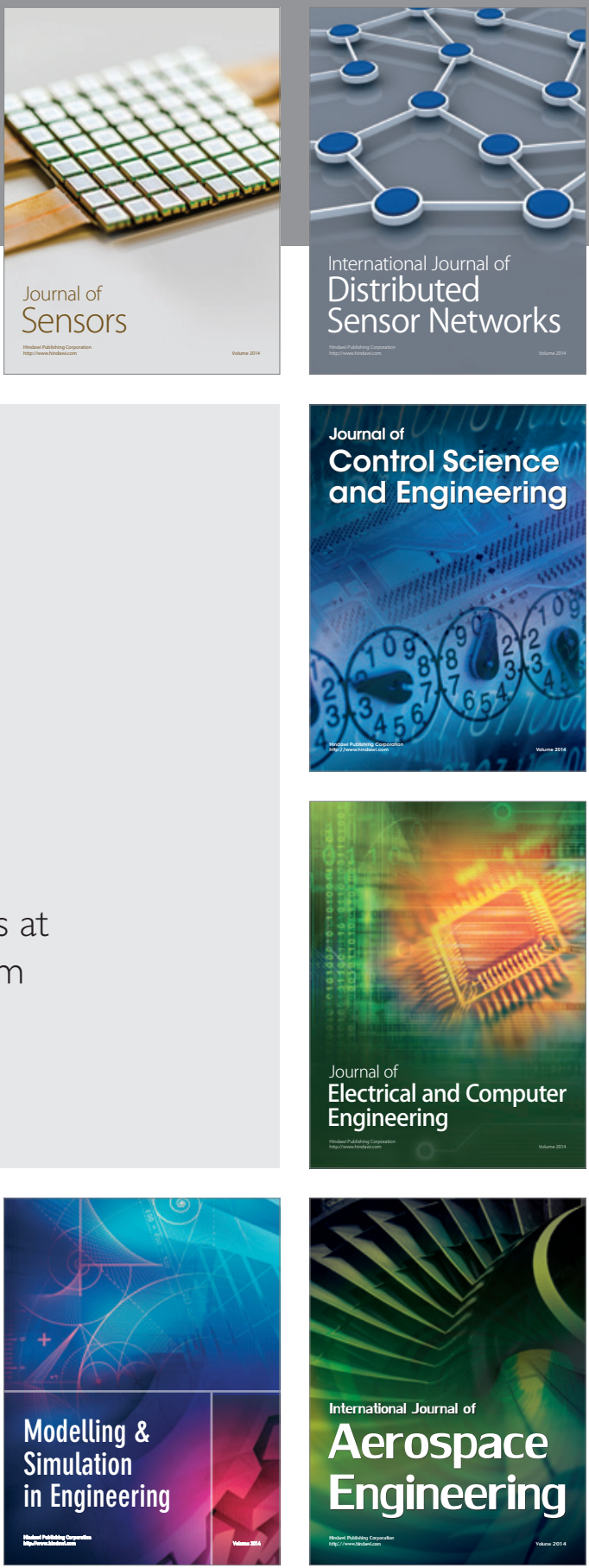

Journal of

Control Science

and Engineering
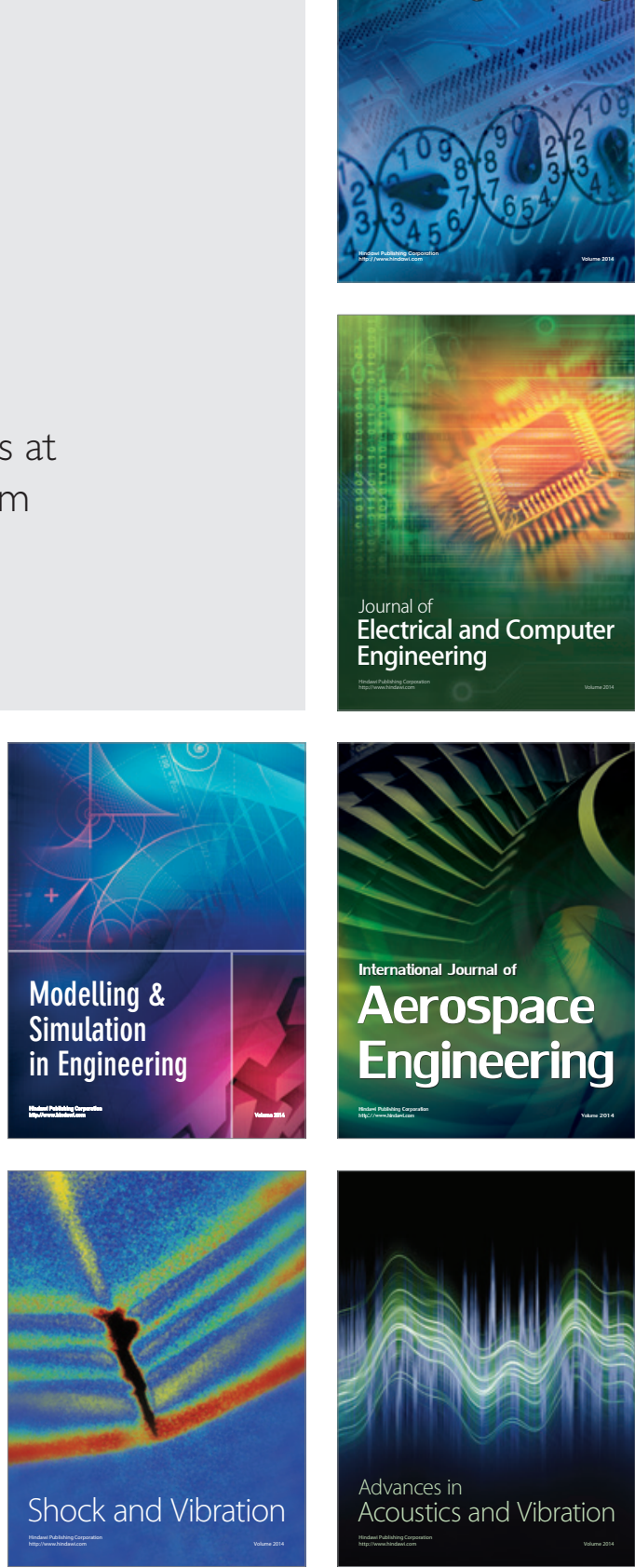